\title{
RECONSTRUCTION AND AFTER-CARE OF OLD UNREDUCED POTT'S FRACTURES *
}

\author{
WILLIAM L. SNEED, M.D. \\ NEW YORK
}

The most frequent disability following in the wake of Pott's fractures is flat foot. This is often exaggerated and rendered painful and persistent by a slight posterior displacement of the foot on the tibia or a widening of the ankle joint, due to a rupture of the tibiofibular ligament. Of course, there is a large percentage of Pott's fractures in which the tibiofibular ligament is not ruptured. These will have a normal ankle joint mortise. There is another factor in the reduction of a flat foot. Often after a Pott's fracture the foot is put up at right angles but in valgus position, and the muscles which support the arch of the foot are put on the stretch, the patient is allowed to walk in the support with the foot in this faulty position and a flat foot develops, the result, largely of loss of muscle tone.

In the treatment of these cases the degree of disability and probable end-results are the main factors to be considered. The age of the patient and the traumatic inflammatory changes, whether vascular, lymphatic, osseous or of the soft parts, should always receive careful attention before operation. The operative procedure varies in each individual case, depending on what is necessary for the establishment of the normal anatomy of the foot. In the after-care, one method may apply to all cases.

I have selected a case for

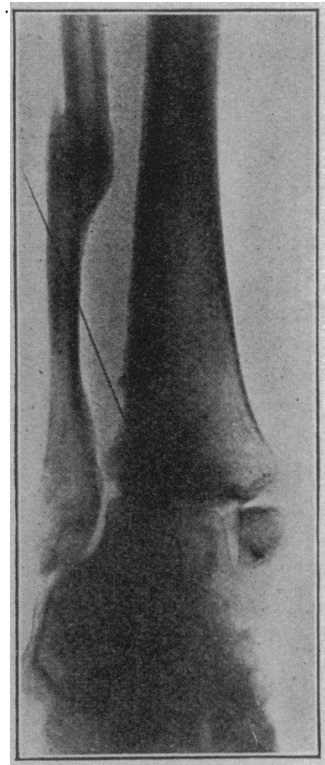

Fig. 1.-Ununited fracture of internal malleolus; marked widening of mortise of ankle joint: rup tured tibiofibular ligament fracture of fibula $31 \%$ inches above joint.

overcorrecting the pes planus and reestablishing the normal relation of the tarsal bones as near as possible.

* Read before the Section on Orthopedic Surgery at the Seventieth Annual Session of the American Medical Association. Atlantic City, N. J., June, 1919 .
Second. An incision was made over the internal malleolus, the fibrous tissue removed, and the ends of the fragments freshened by chiseling. An inlay of bone was slid down from the tibia into a groove prepared in the malleoli, and this was sutured into place.

Third. An incision was made over the fibula and the fibula divided transversely about 1 inch above the ankle joint. Then with a curet the fibrous tissue with

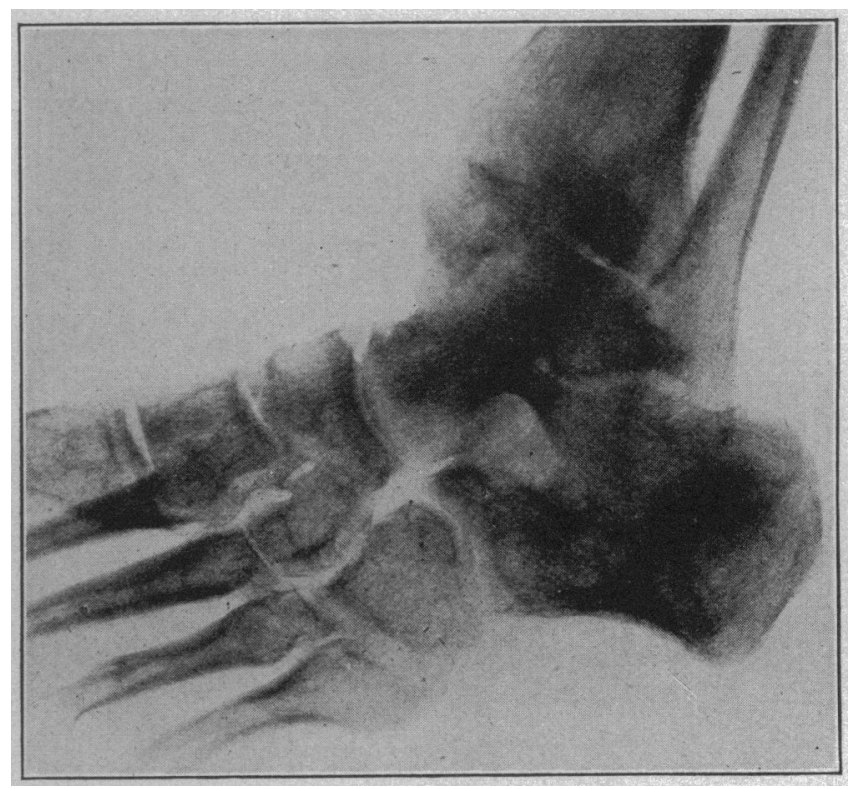

Fig. 2.-Equinus with posterior displacement of foot. The position is equinovalgus.

periosteum was removed from between the tibia and fibula from the ankle joint up to the fracture line.

Fourth. The Achilles tendon was divided by the " $Z$ " method, and the foot was forced up into dorsal flexion. This reduced the posterior displacement. The foot was then pulled around into slight varus, approximating the fracture of the internal malleolus to the tibia and forcing the raw surfaces of the tibia and fibula together. The wounds were then closed.

The foot was put up in plaster of Paris in corrected position and held so for six weeks. A roentgenogram taken at this time revealed a reestablishment of the mortise in the ankle joint by union of the tibia and fibula, a union of the internal malleolus, and an allround reconstruction of the bony anatomy of the foot.

\section{AFTER-CARE}

The after-care consists of : first, continual support; second, baking and massage; third, exercises and manipulations. The support of the foot was of two types: first, plaster of Paris for eight weeks, then adhesive plaster strapping for two or three weeks longer, and finally a Whitman arch support with a raise of shoe of one-quarter inch on the inner border of the heel and toe. The exercises and manipulations are those for reestablishing muscular power of weak feet in general.

In one case, photographs showing the relative contour of the injured and the uninjuced foot reveal less deformity on the injured side, or left foot, than there is on the right side, or uninjured foot. This is accounted for by the forced correction of the flat foot on the injured side while the existing flat foot on the uninjured side was merely supported and not corrected. 
This man returned to work twelve weeks after operation and has been at work since that time, wearing a Whitman brace with his shoe raised one-quarter inch on the inner border of the heels and toes.

\section{OTHER CASES}

I have had two other cases which needed operation. In one only the flat foot and posterior displacement needed correction, necessitating the lengthening of the Achilles tendon. The other patient had a rupture of the tibiofibular ligament. The fibula had healed in a faulty position, and there was a widening of the mortise of the ankle joint. This patient was first seen six weeks after injury. An osteotomy of the fibula and a lengthening of the Achilles tendon, pulling the foot in varus and dorsal flexion, and using the after-care described in the case first mentioned, restored the normal function of this foot, so that the patient has been able to resume his former vocation, which was moving and lifting heavy boxes of goods, without discomfort.

\section{A Caution}

It is very important that the normal mortise of the ankle joint should be restored. If it is not there will be too much play at the ankle joint. The astragalus will be allowed to rotate, and the foot will be permanently weakened. Special stress is laid on this point, as too little attention has been given to this aspect of the lesion both in recent and old cases of Pott's fractures.

\section{ABSTRACT OF DISCUSSION}

Dr. Zabdiel B. Adams, Boston; To reduce these fractures simply means restoring normal conditions as far as possible. The method described originally by Dr. Pott in the treatment of these fractures is not carried out in general. In the classical Pott's fracture the procedures that have been

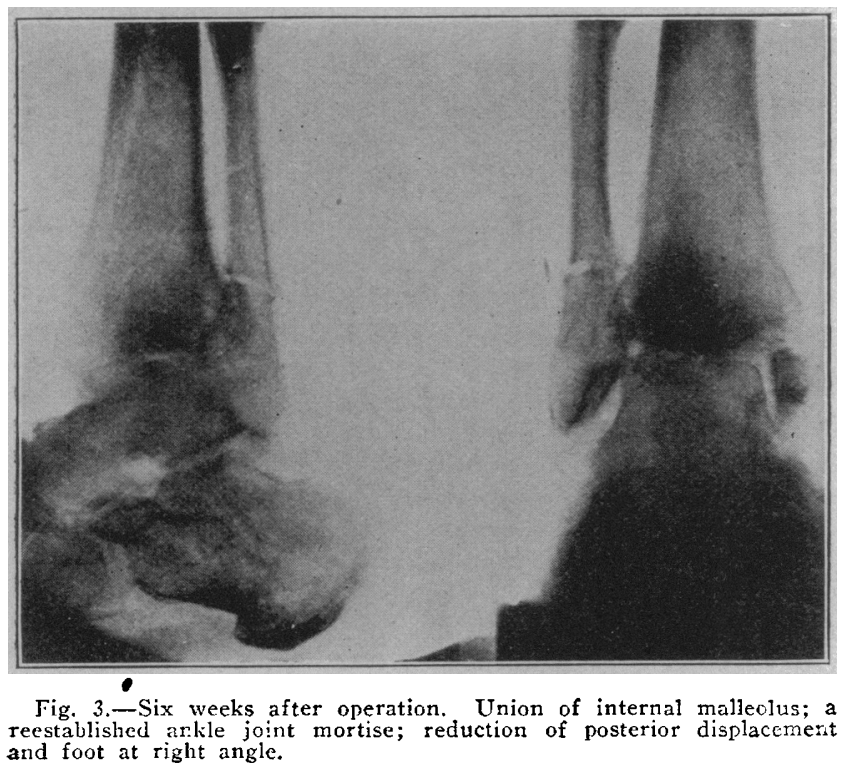
and foot at right angle.

pointed out in this paper are the ones that must be resorted to in order to get a perfect result. If they are carried out, one can, in most of these cases, get a very marked improvement, if not an almost complete cure.

Mr. Ernest W. Hey Groves, England: The keynote of the whole situation here, as in most orthopedic surgery, is prevention. When a Pott's fracture has remained unreduced for any considerable period of time, we can never expect, even by the most ingenious operation, to produce as good a result as if the deformity had been prevented in the first place. So much change takes place in the articular surfaces of the bones, and there is so much play on the ligaments, that a perfect result cannot be looked for. I am quite in agreement, however, with the general lines of treatment laid down by Dr. Sneed.

Dr. Willis C. Campbell, Memphis, Tenn.; The one fact that has been shown in this paper is that these fractures can be rebroken and reduced, the result in many instances being anatomically and functionaliy perfect, without the necessity for any internal splints. Dr. Sneed used, in one instance, the sliding graft, on account of the ununited fracture. The

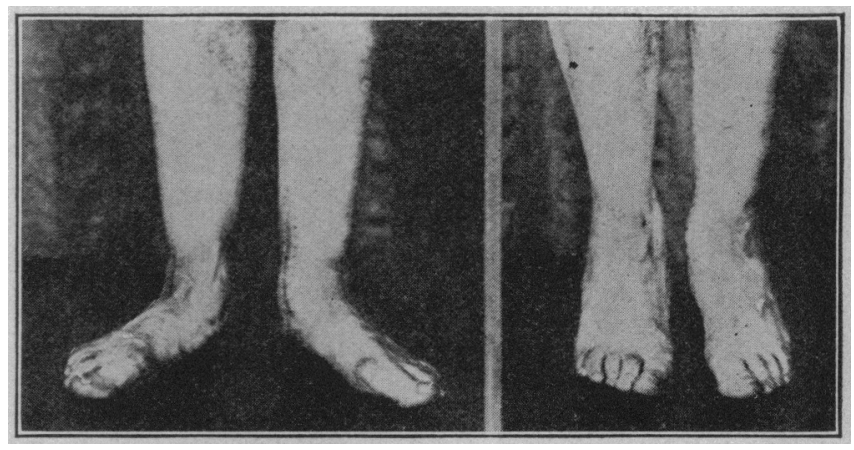

Fig. 4.- Relative contour of both feet eighteen months after operation. Notice long scar over internal malleolus of the left foot and the lesser degree of flat foot on the injured side.

average Pott's fracture is not complicated by nonunion at the point of the mesial fragment, as in his case. Consequently, the raft is unnecessary. I have operated in two cases within the last six months, both being of exactly similar displacement, except that union was perfect. In both cases there was long union between the tibia and the fibula for a considerable distance. This necessitated chiseling between the two bones before replacement. After replacement in proper position, putting the foot in inversion in a plaster cast, I used the simple Thomas heel in shoe, and found it unnecessary to employ any other form of support. Both these cases occurred in young people. The restoration was practically perfect, and I felt that long after-treatment was unnecessary. In old atypical cases, long continued support with the Whitman, or some other form of arch support, would be necessary.

Dr. JAmes W. Sever, Boston: There is one point that I should like to have Dr. Sneed speak of in closing the discussion. That is in regard to the articulation between the tibia and fibula and the rupture of the tibiofibular ligament. In cases in which there is definite injury to the joint, there is a great deal of disability and widening between the two bones. The condition clinically looks like a fracture, but apparently it is not. The adequate treatment of these cases should be discussed, because it is overlooked, the condition being treated as a sprain.

Dr. Harry E. Stewart, New Haven, Conn.: An interesting point has been brought tip about the treatment by means of orthopedic gymnastics and massage. We should not confine a man, after a surgical operation, to an artificial support like a plate, but follow up the procedure with treatment designed to bring back the tone of the muscles and ligaments. We should look on the arch plate as we would on any other splint, to be worn while necessary, and discarded as soon as possible.

Dr. Charles M. Jacobs, Chicago: I consider it a great mistake to immobilize the joint for many weeks as is now being done whether the joint was, or was not, operated on. It is my custom to apply a plaster cast for about ten days; at the end of this time passive motion is used each day for a certain period of time; following which a posterior molded splint is used.

Dr. Fred J. Gaenslen, Milwaukee: Very frequently there is a breaking of the lower and anterior portion of the tibia; and if the fracture is of long standing, this misplaced frag- 
ment acts as a definite obstacle to the reduction of the fracture. In most cases, it would be necessary to remove the fragment or loosen it, so that it would not interfere. Another point is that it is wise to reduce these fractures with the knee in acute flexion. This position relaxes the Achitles tendon avoiding the necessity of tenotomy so that reduction may be accomplished with ease. The plaster may be removed to just below the knee at the end of two weeks, without danger of reluxation.

Dr. William L. Sneed, New York: There is no doubt that many of these cases, as viewed from a roentgen ray standpoint, do not show any fracture. It is possible to have a rupture of the anterior lateral ligament and of the tibiofibular ligament that will give a widening of the joint and injury on the inner side that does not go as far as a fracture, having no bony lesion, but that gives the same symptoms as in Pott's fracture without the deformity, other than a widening of the joint. There is a large percentage of cases in which the tibiofibular ligament is not ruptured. Of course, these cases need no consideration. These patients come to us, maybe one year or two years after the injury, and we must consider them in the light of what can be done at that time, and what it is wise to do, taking into consideration the bony and ligamentous deformity, the muscle degeneration, and the presence or absence of lymp or blood vessel changes. Sometimes it is unwise to do anything at all, as when there is infection in the tarsal bones. I have not known of any case in which it was necessary to carry the support about the knee. We all try to get rid of any mechanical support. If we can restore the normal anatomy, and it needs no support, we do not use it. It is only in the cases in which we cannot restore the normal anatomy that we need to use the support.

\section{ARSPHENAMIN IN PNEUMONIA WITH DELAYED RESOLUTION IN SYPHILITIC SOLDIERS}

\author{
GEORGE DOUGLAS HEAD, M.D. (Minneapolis) \\ Major, M. C., U. S. Army; Chief of the Medical Service, \\ Base Hospital \\ AND \\ JOHN L. SEABLOOM, M.D. (Red OAK, IoWA) \\ Captain, M. C., U. S. Army \\ CAMP WHEELER, MACON, GA.
}

In some individuals an acute disease will so derange the protective mechanism of the body that an old infection lying apparently dormant will be reactivated thereby.

Medical officers stationed during the war in Southern camps where malaria was prevalent not infrequently observed instances of renewed malarial infections in soldiers sick with pneumonia. These soldiers gave old histories of malarial fever with chills, fever and sweats, in some instances years prior to their entrance into the army. The symptoms of malaria appeared usually in the early part of convalescence from pneumonia. The chill and fever were typically periodic. The spleen was usually enlarged. Tertian organisms were found in the blood, and the paroxysms of fever responded promptly to quinin. Several such instances occurred at the base hospital at Camp Wheeler during the epidemic of pneumonia in the winter of 1918-1919.

A severe attack of pneumonia will sometimes weaken or break down the protective cell forces in or about old pathologic lesions in the lung. Pulmonary tuberculosis offers an instance in point. Pneumonia not infrequently lights up an old apparently healed or latent tuberculosis lesion, and the patient who supposed himself cured of tuberculosis is brought face to face in a life and death struggle with his old enemy, the tubercle bacillus.

We are not aware, however, that the attention of the profession has been called to the fact that syphilis may act to prolong or delay the reparative process in the lungs of persons who have had pneumonia, and that syphilitic persons with pneumonia are liable to exhibit signs of delayed resolutions for weeks or months of time. The healing process in these cases does not progress as in the normal individual, and the usual methods of treatment are of no value, while in the cases we have observed, specific treatment has been effective.

The following three cases observed at Camp Wheeler during the epidemic of pneumonia, occurring between Oct. 5, 1918, and Feb. 1, 1919, illustrate the effect of syphilis in causing the delayed resolution of pneumonia and the prompt effect of arsphenamin in clearing it up.

\section{REPORT OF CASES}

CASE 1.-J. J., aged 22, colored, single, soldier in labor battalion, with a negative family history, had measles, mumps and whooping cough as a child, malarial fever in 1914, and smallpox in 1917. He denied having syphilis, but admitted having gonorrhea. Nov. 14, 1918, when going to drill, he developed severe pains in his left side and had a chill. He was admitted to the base hospital the next day with high fever (104), delirium, rapid breathing, dyspnea and marked cyanosis.

The examination of the chest revealed dulness over the entire left lung with bronchial breathing but no râles. A diagnosis of lobar pneumonia of the left lung was made.

From November 14 to the 24 th, the man was very sick with pneumonia, his temperature ranging between 102 and 106.8. He had marked delirium, bloody sputum and high leukocytosis, and a Type IV pneumococcus was isolated from the sputum. November 25, his crisis occurred, and the temperature dropped to normal and remained so until his discharge, March 3, 1919.

In spite of the patient's improved condition, the left lung remained dull to percussion. Crepitant râles and bronchial breathing continued to be heard in the affected area. Roentgen-ray examination revealed an unresolved pneumonia of the left lung. Exploratory puncture of the pleura was made for possible fluid but none was found.

December 30 , six weeks after the onset, the lung showing no signs of clearing, the blood was taken for a Wassermann test, and a ++ report was returned. Since the lung remained consolidated and the usual therapeutic measures had proved of no avail, it was decided to try arsphenamin. The patient was given $0.4 \mathrm{gm}$. intravenously beginning January 14, two months after the onset, and continuing one injection every seven days until five injections had been given. After the second injection of arsphenamin a rapid change in the lung condition took place. The signs of consolidation promptly subsided and the râles disappeared. By February 2, nineteen days after the arsphenamin treatment was started, all signs of lung involvement had cleared.

CASE 2.-James R., aged 25, colored, single, soldier, with a negative family history and with no history of chancre, was admitted to the base hospital, Dec. 5, 1918, complaining of headache, cough, chills and stupor. He stated that three days prior to admission he commenced to feel a pain in his left chest and had a cough. He felt chilly and thought he had fever. On the second day of his sickness he began to cough up some bloody looking sputum and was then sent to the hospital.

The examination on admission revealed nothing of importance outside of the lungs. Over the right lung posteriorly many crepitant râles could be heard on cough or deep breath- 